\title{
Personalized and Translational Medicine in Psychiatry and a Fond Farewell to Wayne Fenton, MD
}

\author{
By Eric Hollander, MD
}

This month's CNS Spectrums includes articles that review topics of special relevance to personalized and translational medicine in psychiatry. In particular, these articles focus on defining endophenotypes (mediating factors between genes and behavior) that can better map onto the etiology and pathophysiology of psychiatric illnesses and identifying genetic polymorphisms that hold the potential to predict response to treatment and side effects in individuals. These highlight emerging themes in psychiatry that hold promise for changing the way physicians and clinicians diagnose mental illness and practice psychiatry in the future.

Before discussing the articles in this issue, I would like to express my personal sense of loss at the tragic death of Wayne Fenton, MD. Wayne was recently murdered by a 19 -year-old patient with schizophrenia seen for an emergency consultation. The patient had refused to take medication, was known to be violent, and needed help. By doing what he felt was in the best interests of this patient and a professional colleague, Wayne placed his own life at risk in order to do the right thing.

Wayne was an administrative leader at the National Institute of Mental Health, often described as Thomas R. Insel, MD's, right-hand man. He was instrumental in shaping many grant initiatives to develop a better understanding of and to treat patients with severe mental illness, and provided sound guidance and mentorship to many investigators, including myself, who trusted his profound insight and his determination to do what was right for the field, and for helping to improve the lives of patients with severe illness. He leaves behind his family, his colleagues, his patients, and his friends, who are all saddened by this loss, as well as a field that is strengthened by his many personal contributions.

We mourn the loss of a leader in the field and remember his many contributions and his determination to do the right thing for his patients and his profession.

In this issue, Maria Alice de Mathis and colleagues note the heterogeneity of obsessivecompulsive disorder (OCD). This variegation has an important impact on various research approaches. One way to delineate OCD subgroups is to search for endophenotypes based on neurophysiological, immunological, genetic, neuropsychological, and neuroanatomic (neuroimaging) paradigms. To identify more homogeneous phenotypic categories, investigators could understand obsessive-compulsive symptom dimensions as quantitative traits and extend the diagnostic boundaries of OCD to include related conditions. While ultimately, the optimal subdivision of categories and subtypes will be based on understanding the etiology of the components of OCD, several solid strategies in combination may provide the best method of subgrouping OCD.

Maria Johnson, RN, and colleagues describe that, to date, the literature has yet to describe a poor metabolizer for cytochromes P450 (CYP) 2D6 and CYP 2 C19. This is rare $(<1 \%$ in the general population) and such individuals are prone to adverse drug reactions with many antidepressants. After genotyping 1,576 participants

Dr. Hollander is the editor of this journal, Klingenstein Professor and Chairman of Psychiatry at Mount Sinai School of Medicine, and director of the Seaver and New York Autism Center of Excellence in New York City. 
of an ongoing pharmacogenetic study in three Kentucky state hospitals, the authors found one poor metabolizer for CYP 2D6 and CYP 2C19. Naturalistic antidepressant treatment of this poor metabolizer for both enzymes is reported. As genotyping reaches clinical practice, it will be interesting to prospectively establish whether specific antidepressants are reasonable choices for these patients, as the scientific data and this case suggest.

William Tucker, MD, and colleagues assessed inmate preferences for in-prison mental health services, outside psychiatric consultants, and telemedicine psychiatric consultation by conducting structured interviews of 28 inmates who received psychiatric telemedicine consultations. Although inmates preferred the use of on-site mental health professionals for many mental health services, some inmates favored visiting or telemedicine consultants for the assessment of safety and sexual issues. Because telemedicine is efficient and readily available, its role in these areas requires further evaluation.

Technology has affected our lives in others ways as well. Elias Aboujaoude, MD, MA, and colleagues investigate the possible negative complications related to Internet use and its place in mental health. In their large-scale epidemiological study of problematic Internet use, the authors surveyed 2,513 adults in the United States. While previous research has suggested the influence and association of other mental illnesses, they discuss the possible classification of problematic
Internet use as an independent mental disorder and its implications. There are many pros and cons to creating a new diagnostic category such as Internet addiction. Contriving a trivial new condition, labeling a human trait, or creating a market for selling pharmaceuticals must be balanced against increasing screening, awareness, and a new understanding and treatment for serious and disabling disorders.

Richard H. Weisler, MD, and colleagues highlight the diverse psychotropic profiles of antiepileptic drugs (AEDs). Some AEDs have proven to be efficacious in the treatment of mood disorders, especially bipolar disorder. Others are ineffective as primary treatments, but may be useful adjuncts for mood disorders or comorbid conditions. Specific AEDs have demonstrated efficacy and Food and Drug Administration indications for the treatment of bipolar patients in acute mania and mixed episodes, and maintenance to delay recurrence. Data for AEDs in acute mania and depression, prophylaxis, and rapid cycling are presented.

This issue unites themes critical to the further elucidation of methods to improve the way researchers, physicians, and clinicians diagnosis, treat, manage, and define the multifactorial needs of the individuals who require our assistance. From genetics and behavior to therapeutic options and classification, these articles contribute to the improvement and development of the myriad aspects of neuroscience. CNS

\section{IN MEMORIAM: PROFESSOR MANFRED ACKENHEIL}

\section{MARCH 14, 1939-AUGUST 1, 2006}

We at CNS Spectrums are also deeply saddened to learn of the untimely passing of
Professor Dr. med. Manfred Ackenheil on August 1, 2006.
A world renowned figure in psychiatric medicine, Professor Ackenheil was at the fore-
front of psychopharmacologic research. His interests and areas of expertise included the
biochemical properties of neuroleptic treatment on schizophrenia patients, and he con-
tributed greatly to the initial research and development of clozapine.
His influence was also felt in his instrumental participation of the organiz-
ing of several important international scientific organizations, such as the American College of
Neuropsychopharmacology and the Collegium Internationale Neuropsychopharmacologicum.
Professor Ackenheil was also the President of the European College of Neuropsychopharmacology
from 1995-1998.
Professor Ackenheil's undisputed skill in and command of the diverse areas of psychiatry were
widely acknowledged during his lifetime.
CNS Spectrums was honored to publish his review on the treatment of neuropathic pain as part of
the April 2005 issue on neuropathic pain.
We extend our sincere condolences to Professor Ackenheil's wife, family, and colleagues.
Joseph Zohar, MD, International Editor

